\title{
Adaptive Anonymous Authentication for Wearable Sensors in Wireless Body Area Networks
}

\author{
Amel Arfaoui $^{*}$, Asma ben Letaifa ${ }^{\dagger}$, Ali Kribeche ${ }^{\ddagger}$, Sidi Mohammed Senouci ${ }^{\ddagger}$, Mohamed Hamdi ${ }^{*}$ \\ *Digital Security Unit, SupCom University of Carthage, Tunisia \\ 'DRIVE EA1859, Univ. Bourgogne Franche Comté, France \\ ${ }^{\dagger}$ MEDIATRON Lab., Sup'Com, University of Carthage, Tunis, Tunisia \\ \{amel.arfaoui, Sidi-Mohammed.Senouci, Ali.Kribeche01\}@u-bourgogne.fr, \{mmh, asma.benletaifa\}@supcom.rnu.tn
}

\begin{abstract}
Wireless body area networks (WBANs) are perceived as an emerging key technology for the next generation ubiquitous healthcare systems. However, the openness and mobility of wireless sensor technologies make the sensor-controller communication vulnerable to be eavesdropped and linked to the sensors in transmission of patient's information. Furthermore, in such resource-constrained environment, authenticating sensor nodes anonymously with the controller node while considering their limited capabilities is a paramount security requirement. In this paper, we propose a lightweight and adaptive anonymous authentication and key agreement scheme for the two-tier WBAN. The proposed protocol enables an anonymous mutual authentication and a session key establishment between the controller node and the body sensor nodes while taking into account the dynamic context changes. From a security perspective, we demonstrate that the proposed key agreement scheme achieves the desired security properties such as anonymity, unlinkability, perfect forward secrecy, etc. Performance analysis proves that the proposed protocol outperforms benchmark schemes in terms of communication and computational overhead.
\end{abstract}

Keywords- WBAN, Anonymous Authentication, Adaptive security, Key agreement, Scyther.

\section{INTRODUCTION}

Wireless Body Area Network (WBAN) is an area field developed in recent years as a result of applying Wireless Personal Area Networks (WPANs) to the communications on, near and around the human body. It is adopted for real-time monitoring of patient's physiological parameters such as heart rate, blood pressure, and temperature. In WBAN, autonomous sensors are deployed to transmit collected data to the controller node, such as Smartphone, tablet, or PDA. Owing to the emerging developments in wireless communication, ubiquitous sensing, sensor miniaturization and digital signal processing, WBAN becomes a promising networking paradigm for the next generation medical IoT systems [1]. However, the open nature of the wireless channel introduces several security threats and impedes its further progress. Therefore, security and privacy are growing concerns in WBAN that require a special attention while considering the body sensors' limited resources and dynamic context changes.

Unlike conventional wireless sensor networks (WSNs), WBAN deals with more critical and sensitive patient health information that imposes various privacy, security and safety concerns. Since the exchanged messages are transmitted through an open channel, they can be eavesdropped, intercepted and tampered. Hence, confidentiality and mutual authentication present an essential security mechanism before data transmission that should be anonymous and unlinkable given that an adversary can't assign or link the transmitted traffic to the same sensor. Furthermore, harsh environmental conditions and stringent resource constraints of body sensors in terms of memory capacity, communication and computational capabilities make security costs become more tangible through energy depletion and performance degradation. In this context, cooperative transmission through relay nodes is used to reduce the communication overhead for sensor nodes that are far from the controller node and extend the network lifetime.

Recently, some contributions have addressed particular attention to the issues of improving security effectiveness, authentication solutions, and key agreement for WBAN.The most obvious application of WBANs is in the medical field, for patient monitoring. Particularly, several research works have turned to implement cryptography-based authentication schemes given that they require fewer constraints related to the applications' environments (channel, distance, and location) than the non-cryptographic methods [2][3][4]. Therefore, they have been attracted a particular attention. On one side, some previous works have focused on the extra-body communication security in WBAN [5] [6] [7] [8]. However, although their security effectiveness, the most of these schemes are considered more as digital signatures than authentication schemes and involve high communication and computation cost. On the other side, some interesting authentication and key agreement schemes for the inter-WBAN communication have been proposed [9] [10] [11] [12]. In [9] [10], the authors presented two authenticated key exchange protocols for two-hop star WBAN topology, which provide a selective authentication between the sensor nodes. At first, an authentication scheme between the sensor nodes and the controller node for normal situations has been proposed. Then, the second scheme is designed for emergency situations where sensor node should communicate and cooperate to analyze and transmit their collected data. However, their protocols don't provide anonymity and sensor nodes communicate with the controller node using their clear identities. Most recently, two anonymous authentication and key agreement schemes for two-tier WBAN have been proposed in [11] [12]. The proposed schemes provide anonymous authentication and session key establishment between the sensor nodes and the hub node. Besides, several security properties have been supported such as unlinkability, backward/forward secrecy and resilience against attacks. However, they can't ensure perfect forward secrecy, which is an essential and basic requirement for a session key based 
authentication protocol. In that case, when an adversary reveals the long-term secret keys, he can deduce any previous session key, which is a serious threat to the real-time data security. In addition, all the aforementioned schemes focus only on the security facet and none of them consider the dynamic context changes of WBAN for relay node selection in order to ensure a tradeoff between energy efficiency and security effectiveness during the authentication and key agreement process.

This study addresses the problem of adaptive anonymous authentication for two-hop WBAN topology where relay nodes should be adaptively selected while considering the dynamic context changes. The main contributions of this paper are as follows:

- A lightweight anonymous authentication and key agreement scheme, which can ensure various security properties like sensor node anonymity, unlinkability, resilience against replay attacks, etc.

- An adaptive relay node selection while considering the dynamic context changes (traffic nature, the channel characteristics, the battery level and the memory capacity).

- The security of the proposed protocol is not only proved through widely accepted BAN logic, but also evaluated by the Scyther simulator tool.

- The efficiency of the key agreement scheme is proven in terms of storage overhead, communication, and computational cost which outperform the benchmarking schemes.

Hence, the remainder of this paper covers the following points: The system model and the context-awareness modeling for adaptive relay node selection are described in Section II. The proposed adaptive anonymous authentication scheme for twotier WBAN is given in section III. Section IV presents the security analysis followed by the performance evaluation. The conclusion is drawn in section VI.

\section{SYSTEM MODEL}

In this section, we describe the network model, the relay node selection decision that impacts the network lifetime as well as the threat model.

\section{A. Network Model}

In this subsection, we present the network topology that provides an efficient security while investigating the relay nodes' deployment. In fact, relay nodes are classified on the basis of their localization, capacity and resources' availability. As a result, the powerful relay node is defined as a primary node, whereas the normal sensor node is termed as a secondary node.

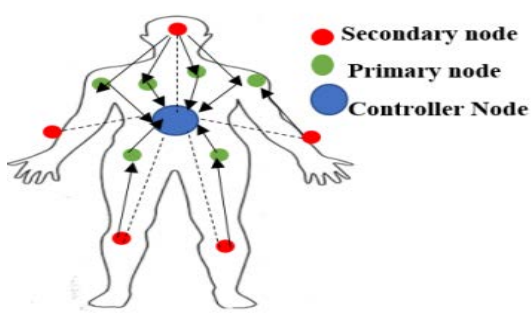

Fig1. WBAN network model
As shown in Fig1, the network model for WBAN is a twotier centralized network, which consists of a controller node and several descendants classified as primary and secondary nodes. The different functions of each entity are presented as follows:

- Controller node $(\mathrm{CN})$ is the center of a BAN network that analyzes the collected data from Intra-BAN and holds the patient's authentication process. In addition, it selects the most appropriate relay node based on the dynamic context changes.

- $\quad$ Secondary Nodes (SN) are resource-constrained wearable sensors attached to the human body. They use the primary nodes to forward real time information to the controller node.

- Primary Nodes (PN) are powerful nodes that have more capacity than normal sensor nodes. They should not only gather physiological vital signs, but also be intermediate nodes between the controller node and the secondary nodes. However, one PN could only be linked with one $\mathrm{SN}$ at a time.

Considering the human body movements, the network topology is in frequent changes. In such case, we assume that if the secondary node is in-range with the controller node, it can conduct direct communication. Otherwise, the controller node selects the most appropriate primary node to rely the traffic while considering the dynamic context changes.

\section{B. Adaptive Relay node selection}

In order to ensure a tradeoff between security effectiveness and energy efficiency, it is essential to select the most appropriate relay node involved in the authentication and key agreement process between the sensor node and the controller node. In this context, we define the dynamic context that affects the selection decision.

\section{1) Context-Awareness Modeling}

Based on the contextual information, each sensor node chooses to communicate directly with the controller node or use a relay node. When a secondary node wants to initiate an authentication session with the controller node, it broadcasts an authentication message. Upon receiving the message from the secondary node, each adjacent primary node generates a message that defines its capacity to be a relay node and send it to the controller node. Subsequently, the controller node computes the cost function of the relay nodes that is dependent on the residual energy, memory capacity and the link quality. Then, it determines the most appropriate primary node to be connected to the secondary node.

\section{a) Energy Model}

To balance energy consumption among sensor nodes and extend the network lifetime, each node considers the residual energy which is presented as follows [13]:

$$
E_{\text {res }, i}=E_{\text {ini }, i}-E_{\text {con }, i}
$$

Where $E_{\text {con }, i}$ is the energy consumed by the node $i$. The energy consumption in a node includes the total amount of transmission and reception energy.

b) Communication Model

The instantaneous SNR between the secondary node and the controller node through a relay node is considered in order to 
determine the most appropriate relay node. The instantaneous SNR is computed as follows [14]:

$$
\gamma_{s, r, C N}=\frac{\gamma_{s, r} * \gamma_{r, C N}}{\gamma_{s, r}+\gamma_{r, C N}+1}
$$

Where $\gamma_{S, r}=\frac{P_{A^{*}}\left|h_{s, r}\right|^{2}}{N_{0}}$ represents the receiver SNR of the relay node; $\gamma_{r, C N}=\frac{P_{R^{*}}\left|h_{r, C N}\right|^{2}}{N_{0}}$ defines the receiver SNR of the controller node from the relay.

\section{c) Memory Model}

We assume that the communication within the BAN is modeled as a queuing process. Therefore, the relay node selection considers the available size of the queue, $Q_{\text {empty }}$, in the relay node.

\section{d) Mobility Model}

In the proposed scheme, we will focus on the dynamic scenario where the body is in motion and the distance between the relay nodes and the controller is dependent on the body movements. The path loss model $P L(d, f)$ is presented as follows [15]:

$$
P L(d, f)=P L_{0}+10 n \log _{10}\left(\frac{d}{d_{0}}\right)+\sigma
$$

Where $P L_{0}=10 \log _{10}\left(4 \pi f d_{0}\right)^{2} * c ; d$ is the distance between the relay and the controller; $d_{0}$ is the reference distance; $n$ is the path loss coefficient; $\sigma$ is the standard deviation; $f$ the frequency of operation; and $c$ is the speed of light.

\section{e) Traffic nature model}

In emergency situations, the secondary node should connect and transmit the collected data directly to the controller. However, in normal conditions, the secondary node communicates with the controller through a relay node.

\section{2) Cost function}

In order to ensure a high energy efficiency and respond to the QoS requirements, the controller node should select the most convenient relay node that has the minimal cost. The cost function $C F(i)$ of an $i^{\text {th }}$ relay node is dependent on its residual energy $E_{\text {res, } i}$, the free queue size $Q_{\text {empty, } i}$ and the communication channel quality and it is given by:

$$
C F(i)=\frac{d}{E_{\text {res }, i} * Q_{\text {empty }, *} * T P_{i}}
$$

Where $d$ is the distance between the relay node and the controller; $T P_{i}=\frac{\gamma_{s, r i, C N}}{\alpha}$ is the transmission power of the relay node $i$ and $\alpha$ is the path-loss component.

\section{Threat Model}

We use the Dovel-Yao threat model as [8] in which two communicating parties interact over an insecure channel. In this model, an adversary can intercept the exchanged messages over the public channel. On one hand, the attacker has the ability to modify, corrupt and delete the content of the transmitted messages communicated over the insecure public channel. On the other hand, he can eavesdrop all the communication links, inject packets and reply old messages previously transmitted.

\section{THE PROPOSED ANONYMOUS AUTHENTICATED KEY AGREEMENT SCHEME}

In this section, we present the proposed adaptive anonymous authentication protocol for the two-tier WBAN. The notations used in our scheme are given in Table 1. In normal situation, if a secondary node is in-range with the controller, it can make direct connection. Otherwise, $\mathrm{CN}$ should find the most suitable primary node based on the contextual information in order to establish the communication. Our proposed scheme consists of two phases: Registration phase and anonymous authentication and key exchange phase. In the first phase, the System Administrator (SA) generates the secure credentials to be stored in the communicating parties' memory. In the anonymous authentication and key exchange phase, both a secondary node and the controller node can establish a session key between them.

\section{A. Notations}

In this subsection, we provide the notations set used in the proposed scheme.

\begin{tabular}{|c|l|}
\hline Notation & \\
\hline$S N$ & Secondary node \\
$P N$ & Primary node \\
$C N$ & Controller node \\
$M K$ & A master key \\
$A I D_{N}$ & One-time alias identity of the node N \\
$I D_{N}$ & Identity of the node N \\
$S I D_{N}$ & Shadow Identity of N \\
$K P_{N}$ & The pre-shared key between the controller and the \\
$T_{S e q}$ & node N The transaction sequence number \\
$S K$ & The session key between SN and CN \\
$h()$. & One-way hash function \\
$T_{E}$ & Time consumed for one exponentiation operation \\
$T_{M}$ & Time consumed for one scalar multiplication on ECC \\
$T_{s y m}$ & Time for one symmetric encryption \\
$T_{H}$ & Time for one hash function \\
$T_{X O R}$ & Time for one XOR operation \\
$T_{A}$ & Time for one addition on ECC \\
$T_{M a p}$ & Time for one map-to-point on ECC \\
$T_{P}$ & Time for one pairing operation. \\
& \\
\hline
\end{tabular}

Table 1. Variables and their descriptions

\section{B. Registration Phase}

In this phase, the system administrator (SA) performs the following steps to register the sensor nodes and the controller before their deployment:

Step 1: Generate a 128-bit secret pre-shared master key $M K$ for all the nodes in WBAN

Step 2: For a sensor node N, assign a unique secret identity $I D_{N}$ Step 3: Compute the pre-shared key between $\mathrm{N}$ and the controller $K P_{N}=\mathrm{h}\left(I D_{N}|| M K\right)$

Step 4: For each sensor node N, the SA executes the following:

- Generate a set of unlikable shadow-IDs $\operatorname{SID}_{N}=\left\{\right.$ sid $_{l}$, $\left.\operatorname{sid}_{2} \ldots\right\}$ where $\operatorname{sid}_{j}=\mathrm{h}\left(I D_{N}\left\|s_{j}\right\| K P_{N}\right)$ and $s_{j}$ is a random number.

- Pick randomly a 64-bit transaction sequence number $T_{\text {seq }}$ Step 5: Store $\left(I D_{N}, S I D_{N}, K P_{N}, T_{\text {seq }}\right)$ into the sensor node's memory and the CN's memory. 
The concept of transaction sequence number is exploited to prevent replay attacks and hasten the authentication process. In fact, the controller can identify the sensor node and verify the legitimacy of the authentication request by comparing the received $T_{\text {seq }}$ with the stored value on its database. Specifically, if the $T_{\text {seq }}$ provided by the sensor node isn't corresponding to the stored value in the controller's database. Then, $\mathrm{CN}$ aborts the connection and the sensor node will use one of its unused shadow identity $\operatorname{sid}_{j} \in S I D_{N}$.

\section{Anonymous Authentication and Key Exchange phase}

This phase performs the anonymous authentication between a secondary node and the controller through the adequate relay node. At the end of this phase, a session key is generated between $\mathrm{SN}$ and $\mathrm{CN}$. The execution of the authentication process consists of the following steps:

Step 1: $\mathrm{SN} \rightarrow \mathrm{PN}\left(M_{l}=\left\{A I D_{S N}, x, V_{l}, T_{\text {seq }}\right\}\right)$

A secondary node $I D_{S N}$ chooses a random number $r$ and performs as follows:

- Generate $A I D_{S N}=\mathrm{h}\left(I D_{S N}\left\|K P_{S N}\right\| r \| T_{\text {seq }}\right)$

- Derive the session key $S K=\mathrm{h}\left(I D_{S N}|| K P_{S N}|| A I D_{S N} \| r\right)$

- Compute $x=r \bigoplus K P_{S N}$

- Compute $V_{l}=\mathrm{h}\left(A I D_{S N}\|S K\| r\right)$

We should notice that, in case of de-synchronization, the sensor node doesn't need to send a transaction sequence number $T_{\text {seq }}$ in $M_{l}$ and it should choose one of its unused shadow-IDs $\operatorname{sid}_{j}$

Step 2: $\mathrm{PN}_{\mathrm{i}} \rightarrow \mathrm{CN}\left(M_{2}=\left\{A I D_{P N i}, y_{i}, M_{l}, T_{\text {seqi }}, S_{i}\right\}\right)$

Upon receiving the authentication message from the secondary node, each adjacent primary node $\mathrm{PN}_{\mathrm{i}}(i=1 \ldots K)$ generates a random number $p_{i}$ and performs as follows:

- Define its current state $S_{i}=\left(E_{\text {res, }, i}, Q_{\text {empty,i },}, \gamma_{S N, P N(i)}\right)$

- Derive $A I D_{P N i}=\mathrm{h}\left(I D_{P N}|| K P_{P N i} \| T_{\text {seqi }}\right)$

- Compute $y_{i}=p_{i} \bigoplus K P_{P N i}$

Step 3: $\mathrm{CN} \rightarrow P N_{i}$ opt $\left(M_{3}=\left\{T_{s}, T_{s i}\right.\right.$ opt $\left.\left., V_{2}, V_{3}\right\}\right)$

- For $i=1 \ldots K$, check $T_{\text {seq }}$

- Check $T_{\text {seq }}$

- Select $P N_{i}$ opt $=\operatorname{argmin} C F(i)$

- Compute $p_{i^{o p t}}^{*}=y_{i^{\text {opt }}}^{*} \oplus K P_{P N_{i} \text { opt }}$

- Check the validity of $A I D_{P N_{i} \text { opt }}$

- Compute $r^{*}=x \oplus K P_{S N}$

- Verify the validity of $A I D_{S N}$

- Compute $S K^{*}=\mathrm{h}\left(I D_{S N}\left\|K P_{S N}\right\| A I D_{S N} \| r^{*}\right)$

- Compute $V_{l}{ }^{*}=\mathrm{h}\left(A I D_{S N}\left\|S K^{*}\right\| r^{*}\right)$ and check $V_{l}{ }^{*}=V_{l}$

- Select randomly $T_{\text {seq }}^{\prime}=m$ and $T_{\text {seqi }}^{\prime}$ opt $=n$

- Compute $T_{S}=\mathrm{h}\left(S K^{*}\left\|I D_{S N}\right\| r^{*}\right) \oplus T_{s e q}^{\prime}$

- Compute $T_{s i}$ opt $=h\left(K P_{P N_{i} \text { opt }}\left\|I D_{P N_{i} \text { opt }}\right\| p_{i^{\text {opt }}}^{*}\right) \oplus T_{\text {seqi }}^{\prime \text { opt }}$

- Compute $V_{2}=\mathrm{h}\left(T_{S}\left\|S K^{*}\right\| I D_{S N}\right)$

- Compute $V_{3}=\mathrm{h}\left(T_{s i}\right.$ opt $\left.\left\|K P_{P N_{i} \text { opt }}\right\| I D_{P N_{i} \text { opt }}\right)$

- Update $K P_{S N}^{+}=h\left(K P_{S N}\left\|I D_{S N}\right\| T_{s e q}^{\prime}\right)$

- Finally, CN sends $M_{3}$ to $P N_{i}$ opt and $\left\{T_{\text {seqi }} \text {, Text }\right\}_{K P_{P N_{i}}}$ to the inadequate primary nodes to notify them about the connection failure.

Step 4: $P N_{i}$ opt $\rightarrow \mathrm{SN}\left(M_{4}=\left\{T_{s}, V_{2}\right\}\right)$
Upon receiving the authentication reply message from $\mathrm{CN}$, $P N_{i^{o p t}}$ computes $\mathrm{h}\left(T_{s i}\right.$ opt $\left.\left\|K P_{P N_{i} \text { opt }}\right\| I D_{P N_{i} \text { opt }}\right)$ and verifies its equality to $V_{3}$. Then, it derives the new transaction sequence number $T_{\text {seqi }}^{\prime}$ opt $=h\left(K P_{P N_{i} \text { opt }}\left\|I D_{P N_{i} \text { opt }}\right\| p_{i^{\text {opt }}}^{*}\right) \oplus T_{s i}{ }^{o p t}$

$\mathrm{PN}_{i}$ opt sends $\mathrm{M}_{4}$ to $\mathrm{SN}$ which performs as follows:

- Compute $\mathrm{h}\left(T_{S} \| S K|| I D_{S N}\right)$ and check if it is equal to $V_{2}$

- Update $T_{\text {seq }}^{\prime}=\mathrm{h}\left(S K\left\|I D_{S N}\right\| r\right) \oplus T_{S}$

- Update $K P_{S N}^{+}=h\left(K P_{S N}\left\|I D_{S N}\right\| T_{s e q}^{\prime}\right)$

Finally, both $\mathrm{SN}$ and $\mathrm{CN}$ store the generated common session for their future communication.

\section{SECURITY ANALYSIS}

In this section, we prove the capacity of the authentication scheme to mitigate several attacks. Then, we verify and analyze the security of the proposed scheme using Burrows-AbadiNeedham (BAN) logic [16] and Scyther tool [17].

\section{A. Security properties and resilience against attacks} Anonymity and unlinkability: From the tuple $<A I D_{S N}, x, V_{l}$, $T_{\text {seq }}>$, we have the one-time alias identity $A I D_{S N}=\mathrm{h}\left(I D_{S N}\left\|K P_{S N}\right\| r \| T_{\text {seq }}\right)$ where the parameter $r$ is chosen randomly and the tuple $<r, T_{\text {seq }}>$ is refreshed in each session. Therefore, there is no relationship between the aliases and the proposed protocol achieves anonymity and unlinkability.

Perfect forward secrecy: In the proposed scheme, after the accomplishment of each transaction both the sensor node and the controller update their shared key $K P_{S N}^{+}=$ $h\left(K P_{S N}\left\|I D_{S N}\right\| T_{\text {seq }}^{\prime}\right)$.Under this assumption, if the adversary compromises $\mathrm{SN}$ or $\mathrm{CN}$, he can manage only $K P_{S N}^{+}$. Since the hash function is one-way, the adversary can't extract $K P_{S N}$ from $K P_{S N}^{+}$.

Eavesdropping attack: The adversary $\mathcal{A}$ can discern the tuple $<A I D_{S N}, x, V_{1}, V_{2}, T_{s}>$. Given that $S K=\mathrm{h}\left(I D_{S N}\left\|K P_{S N}\right\| A I D_{S N} \| r\right)$ and $K P_{S N}=\mathrm{h}\left(I D_{S N}|| M K\right)$ is computed locally in the $\mathrm{CN}, \mathcal{A}$ can't compute $K P_{S N}$. Furthermore, the one-way hash function prevents the adversary to disclose information about the session key from $x, V_{1}, V_{2}, T_{s}$.

Impersonation attack: To impersonate the $\mathrm{CN}$, the adversary needs to compute $K P_{S N}=\mathrm{h}\left(I D_{S N}|| M K\right)$. However, the tuple < $I D_{S N}, M K>$ can't be intercepted from the transmitted messages. For the sensor node, $\mathcal{A}$ has to create a valid tuple $<A I D_{S N}, x, V_{l}$, $T_{\text {seq }}>$. However, he can't figure out the real identity of the sensor from $A I D_{S N}=\mathrm{h}\left(I D_{S N}\left\|K P_{S N}\right\| r \| T_{\text {seq }}\right)$.

Replay attack: the transaction sequence number is used to avoid the replay attack. In the proposed scheme, none of the parameters of the request message $\left\langle A I D_{S N}, x, V_{l}, T_{\text {seq }}>\right.$ can be transmitted twice.

Man-in-the-middle attack: In order to alter $<A I D_{S N}, x, V_{l}, T_{s e q}>$, an adversary $\mathcal{A}$ can generate a random number $r$ ' and try to compute $x$ '. However, he can't determine $x$ ' without $K P_{S N}=\mathrm{h}\left(I D_{S N}|| M K\right)$. For the computation of $S K$, the adversary needs $I D_{S N}$ and $K P_{S N}$ which are secret and unknown to him. Hence, $\mathcal{A}$ can't modify $<A I D_{S N}, x, V_{1}, T_{s e q}>$. The modification of $<T_{s}, T_{s i}$ opt $, V_{2}, V_{3}>$ requires firstly the computation of $V_{2}=$ $\mathrm{h}\left(T_{S}|| S K|| I D_{S N}\right)$ but the adversary can't get anyone of the above 
parameters given that $I D_{S N}$ and $S K$ are secret and $T_{S}$ is computed from them. Then, for $V_{3}=\mathrm{h}\left(T_{s i}\right.$ opt $\left.\left\|K P_{P N_{i} \text { opt }}\right\| I D_{P N_{i} \text { opt }}\right)$, it is impossible to get the secret parameters $K P_{P N_{i} \text { opt }}, I D_{P N_{i} \text { opt }}$ and $T_{\text {si }}$ opt is computed from them.

\section{B. Formal Analysis Based on BAN logic}

In this subsection, we adopt the widely-accepted BAN logic to demonstrate that the proposed authentication protocol ensures secure mutual authentication between a sensor node and the controller. At first, we briefly define the rules of BAN logic [17]. Then, we handle the formal proof.

- R1. Message-meaning rule: $\frac{P \mid \equiv \mathrm{P} \stackrel{Y}{\leftrightarrow} \mathrm{Q}, \mathrm{P} \triangleleft\langle X\rangle_{Y}}{P|\equiv \mathrm{Q}| \sim \mathrm{X}}$

- R2. Nonce-verification rule: $\frac{P|\equiv \#(\mathrm{X}), P| \equiv \mathrm{Q} \mid \sim \mathrm{X}}{P|\equiv \mathrm{Q}| \equiv X}$

- R3. Jurisdiction rule: $\frac{P|\equiv \mathrm{Q}| \Rightarrow \mathrm{X}, P|\equiv \mathrm{Q}| \equiv X}{P \mid \equiv X}$

- R4. Freshness-conjuncatenation rule: $\frac{P \mid \equiv \#(\mathrm{X})}{P \mid \equiv \#(\mathrm{X}, \mathrm{Y})}$

Goals: the main goals of the following analysis are given by:

- $\mathrm{G}_{1}: \mathrm{SN} \mid \equiv \mathrm{CN} \underset{\mathrm{SK}}{\stackrel{S K}{\leftrightarrow}} \mathrm{SN}$

- $\mathrm{G}_{2}: \mathrm{CN} \mid \equiv \mathrm{SN} \stackrel{\mathrm{SK}}{\leftrightarrow} \mathrm{CN}$

\section{Idealized form}

$$
\begin{aligned}
& \mathrm{M}_{1}: S N \rightarrow C N<A I D_{S N}, T_{\text {seq }}, I D_{S N}, S N \stackrel{S K}{\leftrightarrow} C N>_{S N} \stackrel{K P_{S N}}{\longleftrightarrow} C N \\
& \mathrm{M}_{2}: C N \rightarrow S N<I D_{S N}, T_{\text {seq }}, S N \stackrel{S K}{\leftrightarrow} C N>_{S N} \stackrel{K P_{S N}}{\longleftrightarrow} C N \\
& \text { Assumptions }
\end{aligned}
$$

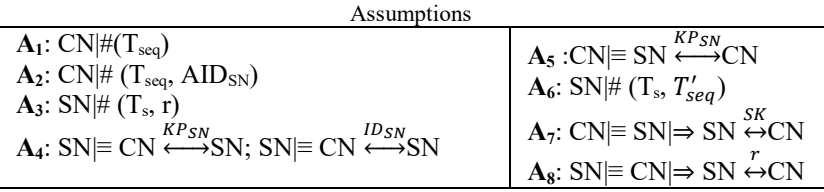

\begin{tabular}{|c|c|}
\hline From $\mathrm{M}_{1}$ & From $\mathrm{M}_{2}$ \\
\hline 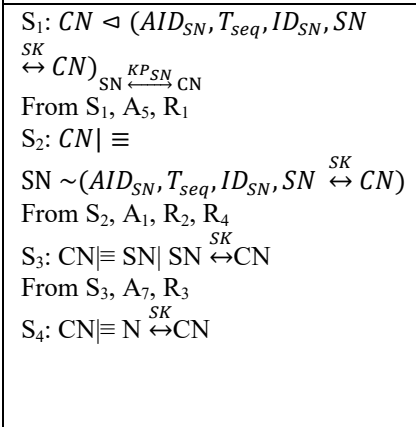 & 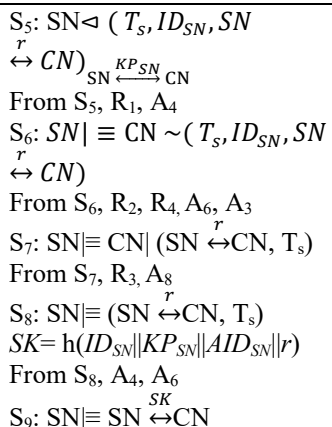 \\
\hline
\end{tabular}

Table 2. List of assumptions of the proposed protocol

Table 3. Security Analysis based on BAN Logic

From Table 3, we conclude that the proposed scheme ensures secure mutual authentication and session key $S K$ establishment between a sensor node $\mathrm{SN}$ and the controller node $\mathrm{CN}$.

\section{Simulation for Formal Security Verification Using Scyther Tool}

In order to evaluate the security of the proposed protocol, we perform an automated security protocol verification tool Scyther which has the ability to test security protocols against several types of attacks. As shown in Fig 2, the verification result indicates that no attacks found on each of the claims specified in our protocol.

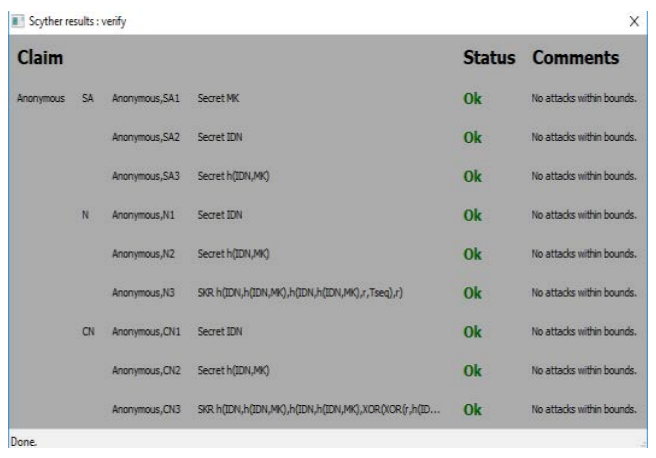

Fig 2. Verification result of the proposed protocol

\section{V.PERFORMANCE ANALYSIS}

In this section, we evaluate the performance of the proposed scheme in terms of storage, communication and computation overhead. At first, we present the storage requirements of the proposed scheme. Then, we compare the communication and the computation costs of the proposed scheme with the benchmarking schemes [5] [6] [7] [9]. For this purpose, we consider the elliptic curve cryptography(ECC) where singular curve $\mathrm{E}\left(F_{p}\right)$ with order $q$ over the finite field $F_{p}$ is used. We assume that $p$ and $q$ are two large prime numbers with 512 and 160 bits, respectively. The lengths of an element in $\mathbb{G}_{1}$, the client's identity, the right, the SHA-1 hash function are 1024, 32, 64, and 160 bit, respectively. To be consistent for the comparisons, we consider a 128-bit AES block cipher $|M|$ for the different benchmarking protocols.

\section{1) Storage overhead}

In the proposed scheme, the controller node is required to store the transaction sequence number $T_{\text {seq }}$, the session key $S K$ as well as the tuple $\left\langle I D_{N}, S I D_{N}, K P_{N}>\right.$ for the registered sensor nodes. Furthermore, each sensor node needs to store the tuple $<$ $I D_{N}, S I D_{N}, K P_{N}, T_{s e q}, S K>$. By applying SHA-1 hash function, we have $\left|I D_{N}\right|=\left|S I D_{N}\right|=\left|K P_{N}\right|=|S K|=160$ bits, while $\left|T_{\text {seq }}\right|=$ 64 bits. Hence, as shown in Table 4, the total storage overhead for the controller node is $544 n+160$, where $n$ is the number of registered sensor nodes. Each sensor node SN stores 704 bits.

\begin{tabular}{|l|l|}
\hline Node & Storage overhead (bits) \\
\hline $\mathrm{N}$ & 704 \\
\hline $\mathrm{CN}$ & $544 \mathrm{n}+160$ \\
\hline
\end{tabular}

Table 4. Storage overhead of the proposed protocol

\section{2) Communication overhead}

In the proposed scheme, $\mathrm{SN}$ needs to broadcast the message $M_{l}=\left\{A I D_{S N}, x, V_{l}, T_{\text {seq }}\right\}$ whose size is 544 bits. Upon receiving $M_{1}$, each concerned $\mathrm{PN}_{\mathrm{i}}$ transmits $M_{2}=\left\{A I D_{P N i}, y_{i}, M_{1}, T_{\text {seq }}, S_{i}\right\}$ to the controller node. Assume $\left|S_{i}\right|=80$ bits, then, the size of $M_{2}$ is $(2 * 160)+544+64+80=1008$ bits. In the transmission $(\mathrm{CN} \rightarrow$ $P N_{i}$ opt $), \mathrm{CN}$ sends the tuple $<T_{s}, T_{s i}$ opt $, V_{2}, V_{3}>$ of size 640 bits. Finally, $P N_{i}$ opt transmits the message $M_{4}=\left\{T_{s}, V_{2}\right\}$ of size 320 bits to SN. As shown in Table 6, the proposed 
authentication scheme has less communication overhead than the benchmarking schemes [5] [6] [7] [9].

\begin{tabular}{|l|l|}
\hline Communicating nodes & Communication overhead (bits) \\
\hline $\mathrm{SN} \rightarrow \mathrm{PN}_{\mathrm{i}}$ & 544 \\
\hline $\mathrm{PN} \rightarrow \mathrm{CN}$ & 1008 \\
\hline $\mathrm{CN} \rightarrow P N_{i^{o p t}}$ & 640 \\
\hline$P N_{i^{o p t}} \rightarrow \mathrm{SN}$ & 320 \\
\hline
\end{tabular}

Table 5. Communication overhead over the different nodes

\begin{tabular}{|l|l|}
\hline Scheme & Communication cost (bytes) \\
\hline Liu et al. -II[5] & $3\left|\mathbb{G}_{1}\right|+$ Hash + MAC $=424$ \\
\hline He[6] & $3\left|\mathbb{G}_{1}\right|+$ MAC + right $|+| I D|+| M \mid=432$ \\
\hline Xiong[7] & $2\left|\mathbb{G}_{1}\right|+9\left|Z_{q}{ }^{*}\right|+|I D|+|M|=456$ \\
\hline Liu et al. [9] & $\begin{array}{l}\text { Protocol-I }: 274 \\
\text { Protocol-II :422 }\end{array}$ \\
\hline Proposed scheme & 314 \\
\hline
\end{tabular}

Table 6. Communication overhead comparisons

\section{3) Computation Cost}

In this subsection, we compare the proposed anonymous authentication scheme with the benchmarking schemes in terms of computational overhead. In this study, we consider the different operations. In the proposed scheme, the controller node executes $9 T_{H}+4 T_{X O R}$. For each sensor node $\mathrm{SN}$, it needs to perform $6 T_{H}+2 T_{X O R}$. Given that $T_{X O R}$ is negligible, the computational costs for $\mathrm{CN}$ and $\mathrm{SN}$ are $9 T_{H}$ and $6 T_{H}$, respectively. To quantify the running time of the cryptographic primitives, we use a 32-bit Cortex- $\mathrm{M}_{3}$ micro-controller at 72 $\mathrm{MHz}$ as [18]. A one-way hash function needs $0.06 \mathrm{~ms}$, whereas an XOR operation takes negligible time. According to [19], the running time of the different operation are $T_{E}=600 T_{H}, T_{M}=$ $72.5 T_{H}, T_{\text {sym }}=T_{H}, T_{A}=13 T_{H}, T_{M a p}=450 T_{H}$ and $T_{P}=1550 T_{H}$.

\begin{tabular}{|l|l|l|}
\hline Scheme & Controller node $(\mathrm{ms})$ & Sensor node(ms) \\
\hline Liu et al. -II[5] & $6 T_{H}+2 T_{\text {sym }}+4 T_{M}+T_{E}+T_{P}=2534$ & - \\
& $T_{H}=152.04$ & \\
\hline He[6] & $4 T_{H}+T_{A}+4 T_{M}+T_{M a p}=757 T_{H}=45.42$ & - \\
\hline Xiong[7] & $11 T_{E}+T_{P}=8150 T_{H}=489$ & - \\
\hline Liu et al. [9] & Protocol-I $: 9.655$ & $\begin{array}{l}\text { Protocol-I : } 9.07 \\
\text { Protocol-II :9.834 }\end{array}$ \\
\hline Proposed scheme & $9 T_{H}=0.54$ & $6 T_{H}=0.36$ \\
\hline
\end{tabular}

Table 7. Computation cost comparisons

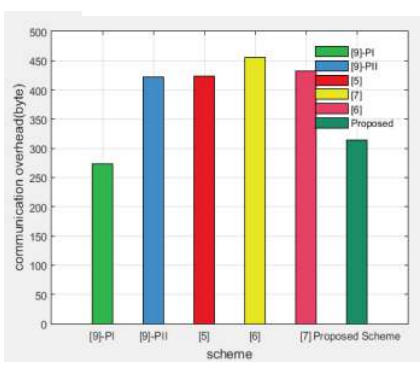

(a)

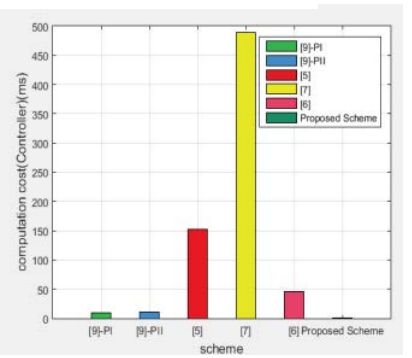

(b)
Fig 3. Communication and Computational overhead comparisons

As shown in Fig 3, the proposed scheme is more efficient than the compared schemes in terms of communication and computational cost.

\section{CONCLUSION AND FUTURE WORK}

In this paper, we proposed a lightweight and adaptive anonymous authentication scheme for inter-WBAN communication. In fact, we considered the dynamic context changes for adaptive relay node selection during the authentication and key agreement process in order to ensure a tradeoff between energy efficiency and security effectiveness. From a security perspective, it has been demonstrated that the proposed authentication and key agreement protocol is secure by using the BAN logic and the simulation tool Scyther. Furthermore, the proposed protocol outperforms the existing schemes in terms of communication and computational overhead. In the future, we aim to provide an adaptive authentication and key agreement scheme for an emergency situation where communication between sensors is required.

\section{REFERENCES}

[1] C. Yi, L. Wang, and Y. Li, "Energy efficient transmission approach for WBAN based on threshold distance," IEEE Sensors J., vol. 15, no. 9, pp. 5133-5141, Sep. 2015 .

[2] Hu, C., Cheng, X., Zhang, F., Wu, D., Liao, X., Chen, D, "OPFKA: Secure and efficient ordered-physiological-feature-based key agreement for wireless body area networks," in Proc. IEEE INFOCOM, 2013, pp. 2274-2282.

[3] Z. Li, H. Wang, M. Daneshmand, and H. Fang, "Secure and efficient key generation and agreement methods for wireless body area networks," in IEEE International Conference on Communications, (ICC), 2017.

[4] T. J. Pierson, X. Liang, R. Peterson, and D. Kotz, "Wanda: securely introducing mobile devices." in Proc. IEEE INFOCOM, 2016.

[5] Liu, J., Zhang, Z., Chen, X., \& Kwak, K. S.," Certificateless remote anonymous authentication schemes for wireless body area networks". IEEE Transactions on Parallel and Distributed Systems, 25(2), pp.332-342, 2014

[6] He, D., Zeadally, S., Kumar, N. Lee, J.H. “Anonymous Authentication for Wireless Body Area Networks with Provable Security”, IEEE Syst. J., 11(4), pp. 25902601,2016 .

[7] Xiong, H., \& Qin, Z. "Revocable and scalable certificateless remote authentication protocol with anonymity for wireless body area networks". IEEE Transactions on Information Forensics and Security, 10(7), pp. 1442-1455, 2015.

[8] P. Gope, T. Hwang, "A Realistic Lightweight Anonymous Authentication Protocol for Securing Real-time Application Data Access in Wireless Sensor Networks," IEEE Transactions on Industrial Electronics, 63(11), pp. 7124-7132, 2016

[9] Liu, J., Li, Q., Yan, R., Sun, R. "Efficient authenticated key exchange protocols for wireless body area networks". EURASIP J. Wirel. Commun. Netw. 2015

[10] J. Liu, Q. Li, R. Sun \& S. Jamal, Energy-efficient key agreement protocols for wireless body area networks, Int. J. Embedded Systems, Vol. 7, 2015

[11] X. Li, M. Ibrahim, S. Kumari, \& al. "Anonymous Mutual Authentication and Key Agreement Scheme for Wearable Sensors in Wireless Body Area Networks, Computer Networks", Vol. 9, pp. 429-443, 2017

[12] M. Ibrahim, S. Kumari, A. Kumar Das, \& al. "Secure anonymous mutual authentication for star two-tier wireless body area networks", computer methods and programs in biomedicine, Vol. 135, pp. 37-51, 2016

[13] V. Ayatollahitafti, M. Ngadi, J. Sharif, M. Abdullahi, "An Efficient Next Hop Selection Algorithm for Multi-Hop Body Area Networks", PLoS ONE11(1): e0146464, Jan.2016

[14] J. Liu, X. Wang, H. Tang, "Physical Layer Authentication Enhancement Using Maximum SNR Ratio Based Cooperative AF Relaying”, Wireless Communications and Mobile Computing, 2017

[15] S. Ahmed, N.Javaid, S.Yousaf, A. Ahmad, M.M. Sandhu, M.Imran, Z.A.Khan, N.Alrajeh, "Co-LAEEBA: Cooperative link aware and energy efficient protocol for wireless body area networks", Computers in Human Behaviors, Vol(51), pp. 1205 1215,2015

[16] M. Burrows, M. Abadi, R. M. Needham, “A logic of authentication”, in: Proceedings of the Royal Society of London A: Mathematical, Physical and Engineering Sciences, volume 426, The Royal Society, 1989, pp. 233-271

[17] Cremers C. "The scyther tool: Verification, falsification, and analysis of security protocols"; International Conference on Computer Aided Verification. Springer; Berlin/Heidelberg, Germany, 2008, pp. 414-418.

[18] J. Liu, Q. Li, R. Yan, R. Sun, "Efficient authenticated key exchange protocols for wireless body area networks", EURASIP J.Wireless Commun. Netw. 2015 (1), pp. $1-11,2015$.

[19] J.-J. Huang, W.-S. Juang, C.-I. Fan, H.-T. Liaw, Robust and privacy protection authentication in cloud computing, Int. J. Innov. Comput. I. 9 (11), pp. 4247-4261, 2013. 